\title{
A comparative study of skin cell activities in collagen and fibrin constructs
}

2 Jia Xian Law ${ }^{\mathrm{a}}$, Faiza Musa ${ }^{\mathrm{c}}$, Ruszymah Bt Hj Idrus ${ }^{\mathrm{a}, \mathrm{b}}$, Alicia J El Haj ${ }^{\mathrm{c}}$, Ying Yang ${ }^{\mathrm{c}, *}$

$3 \quad{ }^{\mathrm{a}}$ Tissue Engineering Centre, Universiti Kebangsaan Malaysia Medical Centre, Jalan Yaacob Latiff, 56000 Kuala Lumpur, Malaysia

$5 \quad{ }^{b}$ Department of Physiology, Faculty of Medicine, Universiti Kebangsaan Malaysia, Jalan Yaacob Latiff, 56000 Kuala Lumpur, Malaysia

$7 \quad$ Institute for Science and Technology in Medicine, School of Medicine, Keele University, Stoke-on-Trent, ST4 7QB United Kingdom

10 Correspondence:

11 Dr. Ying Yang

12 Institute for Science \& Technology in Medicine,

13 Keele University,

14 Stoke-on-Trent,

15 ST4 7QB United Kingdom

16 Phone: +44 (0)1782 674386

17 Email: y.yang@keele.ac.uk

19 Running title: Skin cells culture in different constructs

21 Keywords: Collagen, fibrin, keratinocyte, fibroblast, proliferation, contraction, 22 mechanical property, nanofiber 
Abstract

26 Collagen and fibrin are widely used in tissue engineering due to their excellent

27 biocompatibility and bioactivities that support in vivo tissue formation. These two

28 hydrogels naturally present in different wound healing stages with different regulatory

29 effects on cells, and both of them are mechanically weak in the reconstructed hydrogels.

30 We conducted a comparative study by the growth of rat dermal fibroblasts or dermal

31 fibroblasts and epidermal keratinocytes together in collagen and fibrin constructs

32 respectively with and without the reinforcement of electrospun poly(lactic acid) nanofiber

33 mesh. Cell proliferation, gel contraction and elastic modulus of the constructs were

34 measured on the same gels at multiple time points during the 22 day culturing period

35 using multiple non-destructive techniques. The results demonstrated considerably

36 different cellular activities within the two types of constructs. Co-culturing keratinocytes

37 with fibroblasts in the collagen constructs reduced the fibroblast proliferation, collagen

38 contraction and mechanical strength at late culture point regardless of the presence of

39 nanofibers. Co-culturing keratinocytes with fibroblasts in the fibrin constructs promoted

40 fibroblast proliferation but exerted no influence on fibrin contraction and mechanical

41 strength. The presence of nanofibers in the collagen and fibrin constructs played a

42 favourable role on the fibroblast proliferation when keratinocytes were absent. Thus, this

43 study exhibited new evidence of the strong cross-talk between keratinocytes and

44 fibroblasts, which can be used to control fibroblast proliferation and construct contraction.

45 This cross-talk activity is extracellular matrix-dependent in terms of the fibrous network

46 morphology, density and strength. 


\section{Introduction}

49 Tissue engineering is an emerging multidisciplinary field involving biology, medicine

50 and engineering to restore or regenerate tissue or organ function (1). Tissue engineering

51 consists of 3 core components: cell, scaffold and signaling molecule, that is generally

52 referred to as the tissue engineering triad (2). As one of the main components, scaffold

53 serves as a template for cell delivery and support for tissue remodeling, fills voids and

54 controls the release of signaling molecules. A good scaffold for tissue engineering skin

55 should be biocompatible, biodegradable, support cell growth and tissue regeneration,

56 assists appropriate contraction, and possesses similar mechanical and physical properties

57 as the original skin (3-5). In addition, it is also highly desirable that the scaffold is non-

58 antigenic, non-toxic, readily available, has suitable microstructure, controllable

59 degradation rate and can be stored for a long period of time.

60

61 The scaffold can be made of either natural or synthetic materials. The biggest advantage

62 of natural materials is the excellent biocompatibility that supports cell bioactivities (e.g.

63 attachment, migration, proliferation and differentiation), which in turn regulates and

64 promotes tissue formation. Collagen and fibrin are two of the natural materials that have

65 been widely used in tissue engineering for scaffold fabrication as they fulfill the majority

66 of the desirable characteristics mentioned above. Collagen and fibrin can be easily

67 tailored to form scaffolds that provide proper biological, chemical, structural and

68 mechanical cues to the cells to guide tissue formation in vitro and in vivo $(6,7)$. 
70 Collagen is the major extracellular matrix protein of multiple tissues and organs. For

71 example, approximately $70 \%$ of human skin extracellular matrices is collagen (8).

72 Collagen mainly resides in the dermis, providing mechanical strength to skin (9). To date,

73 more than 29 types of collagen consisted of no less than 46 distinct polypeptide chains

74 have been identified $(10,11)$. Due to its excellent flexibility, collagen has been made into

75 various forms and shapes, including tubes, sponges, sheets, foams, fleeces, nanofibers,

76 and injectable viscous solutions for tissue engineering applications (12).

78 Fibrin is the matrix protein accumulated at wounds after injury to initiate hemostasis and 79 healing (13). Fibrin is formed via the polymerization of fibrinogen monomers in the 80 presence of thrombin. The presence of fibrin as a transitional wound healing matrix

81 during the healing process is crucial, as it has been found to promote haemostasis, 82 angiogenesis, fibroblast proliferation and re-epithelialization, with a potential role in 83 reducing wound contraction and risk of infection (13-16). In addition, fibrin degradation 84 products also have been found to play a profound role in wound healing by inducing 85 fibroblast proliferation, extracellular matrix deposition and angiogenesis (17-19).

87 Collagen and fibrin have been widely used in skin tissue engineering to fabricate tissue88 engineered skin substitutes. However, the two hydrogels have different gelation 89 mechanisms. Fibrin network is initiated by thrombin-catalyzed cleavage of 90 fibrinopeptides from fibrinogen to form fibrils. Collagen fibrils formation is through 91 fibrillogenesis by self-assembly of triple-helical protocollagen molecules. Thus, the 92 collagen fibers exhibit characteristic long bundling with twisted networks, whilst the 
93 fibers in the fibrin appear straighter and more individual (20). Furthermore, collagen and

94 fibrin gels have low mechanical properties initially, and collagen tends to contract,

95 resulting in slower tissue regeneration and less favorable scar quality upon healing.

96

97 In contrast to natural materials, mechanical properties, microstructure and degradation

98 time of synthetic polymers can be easily tailored and controlled to meet the requirement

99 (21). However, synthetic polymers lack cell-recognition signals. This undesirable

100 characteristic can be altered via the addition of chemical functional groups on the

101 polymer surface (22). Another easier and probably more common alternative is the

102 mixing of synthetic and natural materials. The combination of the advantages of both 103 materials renders it more suitable for tissue engineering applications.

105 In this study, we intent to compare the regulatory effect of two hydrogels, collagen and 106 fibrin, on skin regeneration and also the regulatory effect of keratinocytes on fibroblasts 107 when grown in a different matrix environment. The poly(lactic acid) (PLA) nanofibers 108 were incorporated into the collagen and fibrin constructs to improve their mechanical 109 properties. We hypothesize that such a comparison study of the comprehensive matrix 110 combination will provide a valuable communication for better selection of scaffolds in 111 skin generation.

112

113 Materials and methods

114 Isolation and culture of murine epidermal keratinocytes and dermal fibroblasts 
115 Murine dermal fibroblasts and epidermal keratinocytes were isolated using a method 116 described previously (23). In brief, the skin from 4-6 month-old Sprague-Dawley rats was

117 cleaned from fats and hairs before cutting into $1-2 \mathrm{~mm}^{2}$ pieces. The rats were killed by 118 approved Schedule 1 methods, following guidelines from the UK Animals, Scientific 119 procedures Act, 1986 and authorization from Keele Universities’s local ethics committee.

120 Then, the sample was digested with $0.6 \%(\mathrm{v} / \mathrm{w})$ collagenase type I (Sigma, USA) at $37^{\circ} \mathrm{C}$ 121 for 2-3 hours under constant agitation, followed by $0.05 \%$ (w/v) trypsin-EDTA (TE; 122 Lonza, Belgium) for 10 minutes to dislodge the cells. Isolated cells were cultured in 123 Epilife medium (Gibco, UK) and F12:DMEM medium (Gibco) supplemented with 15\% 124 fetal bovine serum (FBS; Lonza) at equal volume. The cells were cultured at $37^{\circ} \mathrm{C}$ and $1255 \% \mathrm{CO}_{2}$. The medium was changed three times per week. Upon $80 \%$ confluence, 126 fibroblasts were separated by exposing the culture to TE for 4 minutes. Separated 127 fibroblasts were cultured with F12:DMEM medium supplemented with 15\% FBS, 128 whereas remaining keratinocytes were cultured with Epilife medium.

130 Electrospinning of PLA nanofibers

131 A 2\% PLA solution was prepared by dissolving PLA (Sigma) in chloroform (Sigma) and 132 dimethylformamide (Sigma) in ratio 7:3. The process of electrospinning follows the 133 established protocol (24). In detail, the PLA solution was placed in a $10 \mathrm{ml}$ glass syringe 134 fitted with 18G blunt end stainless steel needle. Random nanofibers were collected using 135 round stainless steel wire ring of diameter $9 \mathrm{~cm}$. Electrospinning was performed using the 136 following processing parameters: $\pm 6 \mathrm{kV}, 18 \mathrm{~cm}$ air gap, $0.025 \mathrm{ml} / \mathrm{min}$ flow rate and 0.200 
137 ml volume. Collected nanofibers were air dried overnight and sterilized by UV radiation 138 for 3 times, 90 seconds each, before use.

140 Preparation of collagen and fibrin constructs

141 Collagen constructs were prepared using $3.6 \mathrm{mg} / \mathrm{ml}$ rat tail collagen type I solution (BD

142 Bioscience, USA). A total of $0.5 \times 10^{6}$ fibroblasts (F, labeled with PKH 2, green

143 fluorescence (Sigma)) were seeded per construct. The components of the final collagen 144 constructs were 83.33\% collagen type I solution, 10\% 10× DMEM, $1.92 \% 1 \mathrm{~N} \mathrm{NaOH}$ 145 and $4.75 \% \mathrm{dH}_{2} \mathrm{O}$. The final collagen concentration was $3 \mathrm{mg} / \mathrm{ml}$. Collagen constructs 146 were formed by placing $0.5 \mathrm{ml}$ collagen mixture solution on top of a hollow filter paper 147 ring of diameter $25 \mathrm{~mm}$ to prevent lateral contraction. To prepare collagen constructs with 148 random nanofibers (NF), $0.1 \mathrm{ml}$ of collagen gel mixture was used to form the base before 149 random PLA nanofibers were placed on top of it and sealed with $0.4 \mathrm{ml}$ collagen gel 150 mixture (S1). The collagen mixture construct was incubated at $37^{\circ} \mathrm{C}$ for 45 minutes for 151 complete gelation before F12:DMEM medium supplemented with 15\% FBS, 1\% 152 antibiotic-antimycotic (AA; Gibco) and $50 \mu \mathrm{g} / \mathrm{ml}$ ascorbic acid was added. 153

154 Fibrin constructs were prepared using human plasma fibrinogen (Calbiochem, USA). The 155 final fibrin constructs contained $5 \mathrm{mg} / \mathrm{ml}$ fibrinogen, $1 \mathrm{U} / \mathrm{ml}$ thrombin (Calbiochem) and $1562 \mathrm{mg} / \mathrm{ml}$ aminocaproic acid (ACA, Sigma). Each fibrin construct consisted of $0.5 \mathrm{ml}$ 157 fibrin solution with $0.5 \times 10^{6}$ fibroblasts (labeled with $\mathrm{PKH} 2$ ). Fibrin constructs were 158 formed by placing $0.5 \mathrm{ml}$ fibrin gel solution on top of a hollow filter paper ring. To 159 prepare fibrin construct with NF, NF were placed on top of filter paper ring, followed by 
$1600.5 \mathrm{ml}$ fibrin solution. Fabricated constructs were incubated at $37^{\circ} \mathrm{C}$ for 1 hour before 161 F12:DMEM medium supplemented with 15\% FBS, 1\% AA, $50 \mu \mathrm{g} / \mathrm{ml}$ ascorbic acid and 2

$162 \mathrm{mg} / \mathrm{ml}$ ACA was added. ACA is a lysine analog that promotes rapid dissociation of 163 plasmin and is thus an inhibitor of fibrinolysis. $1 \times 10^{5}$ keratinocytes (K; labeled with PKH 164 26, red fluorescence (Sigma)) were seeded on top of the collagen and fibrin constructs on 165 day 2. Fabricated constructs were cultured at $37^{\circ} \mathrm{C}$ and $5 \% \mathrm{CO}_{2}$ with medium changed 166 every 3 days.

167

168 For both hydrogels, four groups of samples have been constructed respectively as 169 indicated in follows: $\mathrm{NF}^{-} \mathrm{K}^{-}$: constructs with fibroblasts but without nanofibers and 170 keratinocytes; $\mathrm{NF}^{-} \mathrm{K}^{+}$: constructs with fibroblasts and keratinocytes but without 171 nanofibers; $\mathrm{NF}^{+} \mathrm{K}^{-}$: constructs with fibroblasts and nanofibers but without keratinocytes 172 and $\mathrm{NF}^{+} \mathrm{K}^{+}$: constructs with fibroblasts, keratinocytes and nanofibers

173

174 Labeling of fibroblasts with PKH 2 and keratinocyte with PKH 26 were performed 175 according to manufacturer's recommendation. In brief, trypsinized cells were washed in 176 serum-free medium before suspended in $300 \mu \mathrm{l}$ of Diluent $\mathrm{C}$ and $300 \mu \mathrm{l}$ of $4 \mu \mathrm{M} \mathrm{PKH}$

177 dye (for the staining of $6 \times 10^{6}$ cells). The cells were incubated in dark for 10 minutes and 178 washed 3 times before mixing with the gels for construct fabrication.

180 Confocal microscopy

181 Three-dimensional fluorescence imaging of the collagen and fibrin constructs was 182 performed to observe the cell distribution after the samples were fixed with 
183 paraformaldehyde (Sigma) overnight. Images were captured at XYZ-axis (20 $\mu$ m step 184 size in Z-axis) using a 10x dry objective lens. The 3-D image was built by stacking of the 185 captured 2-D images.

187 Cell proliferation

188 The number of cells within the cultured constructs at selected days was determined using 189 alamarBlue ${ }^{\circledR}$ cell viability reagent. In brief, spent culture medium was replaced with fresh 190 culture medium with $10 \%$ alamarBlue ${ }^{\circledR}$ reagent. The cultures were incubated at $37^{\circ} \mathrm{C}$ for 1913 hours before fluorescence measurement was performed using a microplate reader with 192 fluorescence excitation wavelength of $530 \mathrm{~nm}$ and the fluorescence emission was read at 193 wavelength $590 \mathrm{~nm}$.

195 Mechanical testing

196 Mechanical testing was performed using non-destructive ball indentation technique that 197 allows time series analysis as described previously (25). In brief, constructs were 198 circumferentially clamped in between 2 transparent plastic circular rings that were held in 199 place by tightly screwed thin stainless steel plates (Figure 1A). The constructs were 200 deformed by placing a PTFE sphere of weight $0.072 \mathrm{~g}$ and diameter $4 \mathrm{~mm}$ in the centre. 201 Images of deformed constructs were acquired 5 minutes after placing the sphere at day 1 , 202 4, 10 and 22 using a long working distance microscope system. The extent of the 203 deformation $(\delta)$ was measured using Image J. 
205 The cross-sectional thickness of each construct was measured using a home-built optical 206 coherence tomography (OCT) (26). Construct contraction was measured as changes in

207 thickness as the filter paper ring prevented contraction in all other directions. Thickness

208 measurement was performed at day 1, 4, 10 and 22.

209

210 Illustration of hydrogel indentation by a sphere is shown in Figure 1B. Elastic modulus (E)

211 was calculated using the following mathematical equation (27);

$212 \quad 6 \mathrm{wr}=\operatorname{Eh}(0.075 \delta 2+0.78 \mathrm{r} \delta)$

213 where $\mathrm{h}$ is the construct thickness, $\mathrm{r}$ is the radius of the sphere and $\mathrm{w}$ is the weight of the

214 sphere. The measurement was ensured that the ratio of a/r was equal to 5 and $\delta / \mathrm{r}$ below

215 1.7, where a was the radius of the clamped portion of the construct to meet the equation

216 condition. This model also assumes that the ratio of thickness to the radius is low and the

217 deformation is large, hence, stretching of the membrane dominates over bending.

218

219 Statistical analysis

220 The data are presented as mean \pm SEM $(n=3)$ and analyzed using Statistical Package for 221 Social Science (SPSS, version 20.0). Statistical analysis was performed using one-way 222 analysis of variance (ANOVA). The differences were considered significant if $\mathrm{p}<0.05$.

224 Results

225 Dermal fibroblast and epidermal keratinocyte isolation and culture

226 Murine dermal fibroblasts and epidermal keratinocytes were isolated via the sequential 227 treatment with collagenase type I and trypsin-EDTA. Culture of the isolated cells showed 
228 the presence of spindle-shaped fibroblasts and cobblestone-shaped keratinocytes (Figure 229 2A). Upon confluence (approximately 8-10 days), the keratinocytes formed colonies that

230 were surrounded by fibroblasts (Figure 2B). The co-cultured cells were separated via

231 differential trypsinization upon reaching $80 \%$ confluence to yield highly pure fibroblasts 232 and keratinocytes (Figure 2C \& D).

233

234 Fabrication of collagen and fibrin-based skin constructs

235 Collagen and fibrin constructs were fabricated with a round filter paper ring as support to 236 prevent lateral contraction. This was important to maintain the shape and size for 237 mechanical testing. Grossly, the collagen and fibrin constructs looked similar with 238 smooth flat surface and translucent appearance at the early culture time point (Figure 3). 239 Nanofibers within the collagen and fibrin constructs could not be seen due to the 240 hydrogels' translucent characteristic and the low density of fine PLA nanofibers. The 241 fiber diameter was measured ranging from 500-800nm and fiber line density of $242182 \pm 8 / \mathrm{mm}$ (S2). To tract the cell migration within the constructs, fibroblasts and 243 keratinocytes were labeled with $\mathrm{PKH} 2$ (green) and PKH 26 (red), respectively. 244 Keratinocytes did not migrate into the fibroblast layer as shown by the presence of a layer 245 of red fluorescent keratinocytes on top of the green fluorescent fibroblasts after 22 days of 246 culture (Figure 4).

\section{Cell proliferation}

249 Cell proliferation was examined via alamarBlue ${ }^{\circledR}$ assay. For the constructs without 250 nanofibers, comparison between fibroblast only groups showed that the fibroblasts 
251 number was significantly higher in the $\mathrm{NF}^{-} \mathrm{K}^{-}$collagen construct compared to the $\mathrm{NF}^{-} \mathrm{K}^{-}$

252 fibrin construct at day 4 and 22. After 22 days in culture, the fibroblast number almost

253 double in the $\mathrm{NF}^{-} \mathrm{K}^{-}$collagen construct, whereas the fibroblast number in the $\mathrm{NF}^{-} \mathrm{K}^{-}$

254 fibrin construct maintained the same. For the constructs with keratinocytes, the $\mathrm{NF}^{-} \mathrm{K}^{+}$

255 collagen construct has significantly higher cell number (keratinocytes+fibroblasts)

256 compared to the $\mathrm{NF}^{-} \mathrm{K}^{+}$fibrin construct at day 4, but the opposite were detected at day 10

257 and 22. The cell number in the $\mathrm{NF}^{-} \mathrm{K}^{+}$collagen construct gradually reduced with time,

258 whereas the cell number in the $\mathrm{NF}^{-} \mathrm{K}^{+}$fibrin construct increased dramatically (Figure 5A).

259

260 Analysis of the constructs with nanofibers showed that the $\mathrm{NF}^{+} \mathrm{K}^{-}$fibrin construct 261 contained more fibroblasts compared to the $\mathrm{NF}^{+} \mathrm{K}^{-}$collagen construct at day 10 , but the

262 opposite was detected at day 22. The $\mathrm{NF}^{+} \mathrm{K}^{+}$fibrin construct has significantly more cells

263 compared to the $\mathrm{NF}^{+} \mathrm{K}^{+}$collagen construct at day 10 and 22 . The cell number in all the 264 groups increased by 2-3 times after 22 days in culture, except the $\mathrm{NF}^{+} \mathrm{K}^{+}$collagen 265 construct (Figure 5B).

266

267 Cell proliferation pattern was different between the collagen and fibrin constructs. The $268 \mathrm{NF}^{-} \mathrm{K}^{-}$and $\mathrm{NF}^{+} \mathrm{K}^{-}$collagen constructs demonstrated slow cell proliferation from day 4 to 269 day 10 and accelerated cell proliferation from day 10 to day 22. In contrast, cell 270 proliferation of the $\mathrm{NF}^{-} \mathrm{K}^{+}, \mathrm{NF}^{+} \mathrm{K}^{-}$and $\mathrm{NF}^{+} \mathrm{K}^{+}$fibrin constructs was fast from day 4 to day 27110 but slowed down from day 10 to day 22 .

272

273 Construct contraction 
274 Construct contraction was measured in term of changes in thickness compared to day 1 275 using a home built OCT (S3 and S4). For the constructs without nanofibers, the collagen

276 constructs showed a reduction in thickness, whilst fibrin constructs' thickness either no

277 change or slightly increased. At day 4, significant different were detected between the $278 \mathrm{NF}^{-} \mathrm{K}^{-}$collagen construct with the $\mathrm{NF}^{-} \mathrm{K}^{-}$and $\mathrm{NF}^{-} \mathrm{K}^{+}$fibrin constructs, and the $\mathrm{NF}^{-} \mathrm{K}^{+}$ 279 collagen construct with the $\mathrm{NF}^{-} \mathrm{K}^{+}$fibrin construct. At day 10 and 22, the thickness of the $280 \mathrm{NF}^{-} \mathrm{K}^{-}$collagen construct reduced significantly more compared all the other constructs. In 281 addition, the $\mathrm{NF}^{-} \mathrm{K}^{+}$collagen construct also showed a significantly higher reduction in 282 thickness compared to the fibrin constructs (Fig. 6A).

284 All the constructs with nanofibers demonstrated a slight reduction in thickness except for 285 the $\mathrm{NF}^{+} \mathrm{K}^{-}$collagen. Significant differences were detected between the $\mathrm{NF}^{+} \mathrm{K}^{-}$collagen 286 construct compared to all the other constructs at day 22 (Fig. 6B). Generally, the $\mathrm{NF}^{-} \mathrm{K}^{-}$ 287 and $\mathrm{NF}^{+} \mathrm{K}^{-}$collagen constructs' thickness reduced gradually with time, whereas all the 288 others groups showed less change with time.

290 Mechanical property

291 Calculation of the elastic modulus showed that incorporation of nanofiber into the 292 collagen and fibrin constructs insignificantly increased the mechanical strength. In all the 293 experimental groups, only the $\mathrm{NF}^{-} \mathrm{K}^{-}$and $\mathrm{NF}^{+} \mathrm{K}^{-}$collagen constructs demonstrated 294 significant increased in Young's modulus with time, whereby significant differences were 295 detected between day 22 and all the earlier time points (Figure 7A \& B). Comparison 296 between the fibrin constructs showed that the presence of keratinocytes and nanofiber 
297 mesh exerted no influence on the construct mechanical strength. For the collagen

298 constructs, it was found that constructs without keratinocytes were significantly stronger

299 compared to the constructs with keratinocytes regardless of the presence of nanofibers at

300 later culturing period.

301

\section{Discussion}

303 Both fibrin and collagen play an important role in wound healing. Fibrin is the 304 provisional matrix at the early phase of healing, replaced by collagen at the later phase for

305 scar tissue formation. Although both collagen and fibrin have been used as a scaffold for

306 skin tissue engineering, there was no systematical or comparison report of their different 307 regulatory effect on skin cells, also the regulatory effect on the cellular cross-talk when 308 keratinocytes and fibroblasts were co-cultured. The current comparative study has 309 generated interesting data by multiple non-destructive techniques and demonstrated that 310 hydrogel scaffolds can exert considerable influence on the skin cells and their cross-talk 311 activities.

312

313 The alamarBlue measurements indicated that collagen and fibrin have different influences 314 on cell proliferation. For the cell proliferation, comparisons were made between the 315 collagen and fibrin constructs that only contained fibroblasts and also between the 316 collagen and fibrin constructs that contained both fibroblasts and keratinocytes. It was 317 found that collagen and fibrin constructs supported and suppressed fibroblast proliferation, 318 respectively, in the absence of keratinocytes. However, keratinocyte and fibroblast co319 culture suppressed cell proliferation in collagen constructs but promoted the cell 
320 proliferation in fibrin constructs. The presence of nanofibers did not alter the cell

321 proliferation activities as the same cell proliferation pattern was seen in the nanofiber

322 incorporated constructs. These findings showed that the fibroblast proliferation in 3-D

323 lattices and keratinocytes' regulatory effect on fibroblast proliferation was matrix

324 dependent. Similarly, Eisinger et al. showed that epidermal cell-derived factors suppress

325 fibroblast proliferation in collagen lattice and Sese et al. found that co-cultured

326 keratinocytes and fibroblasts in fibrin construct proliferated faster than when they were

327 incorporated alone $(28,29)$. These observations reminiscent the natural wound healing

328 process whereby keratinocytes secrete soluble factors that promote fibroblast proliferation

329 during wound healing (wound bed rich in fibrin) and reduced the fibroblast number after

330 healing (wound bed rich in collagen). We speculated that matrix proteins may influence

331 the keratinocyte-mediated regulation of fibroblast proliferation. Furthermore, the

332 alamarBlue data indicated that nanofibers increased the fibroblast proliferation in both

333 collagen and fibrin constructs without keratinocytes. PLA nanofibers may provide

334 mechanical or chemical cues to the fibroblasts to stimulate proliferation.

335

336 Thickness measurements showed that all the collagen constructs demonstrated reduced

337 thickness, whereas the fibrin constructs without nanofibers increased in thickness and

338 those with nanofibers decreased slightly in thickness. Keratinocyte-fibroblast co-culture

339 reduced the collagen constructs' contraction. Previous studies by Chakrabarty et al. (30)

340 and Isaac et al. (31) showed that keratinocyte-fibroblast co-culture increased collagen

341 contraction. Furthermore, Souren et al. showed that co-existentance of keratinocytes and

342 fibroblasts on top and in the collagen lattice, respectively, resulted in greater contraction 
343 compared to the separate presence of both cell types (32). However, consistent with our

344 results, Eisinger et al. found that epidermal cell-derived factor inhibits collagen

345 contraction (28). The lower collagen contraction in our study was probably due to the

346 suppression of fibroblast proliferation, migration and reduction of traction forces by the

347 cytokines secreted by keratinocytes, supported by the findings of lower cell number in the

348 co-cultured collagen constructs. Nien et al. found that fibrin appeared to inhibit

349 contraction of matrix, which might explain our observation (33). Whether the fibrin could

350 exert hypertrophic effects on the fibroblasts is not yet known. Interestingly, the presence

351 of nanofibers in fibrin construct with or without keratinocytes triggered small amount

352 contraction, implying that fibroblasts adhered to nanofiber rather than in fibrin could 353 override the fibrin inhibition influence.

355 Mechanical testing showed that highly contracted constructs were mechanical stronger 356 compared to the less contracted counterparts, as shown by the hike in Young's modulus 357 of highly contracted $\mathrm{NF}^{-} \mathrm{K}^{-}$and $\mathrm{NF}^{+} \mathrm{K}^{-}$collagen constructs at day 22. Incorporation of 358 nanofiber mesh insignificantly improved the Young's modulus of both collagen and fibrin 359 constructs, probably due to the low density nature of the incorporated mesh. Several 360 strategies can be used to improve the mechanical properties of the construct through PLA 361 nanofiber mesh, including reinforcement with denser nanofiber mesh, increasing of the 362 diameter of incorporated nanofibers, substituting random nanofibers with aligned 363 nanofibers and crosslinking of nanofiber mesh (34-37). Nonetheless, each option has its 364 own disadvantages, crosslinking and dense nanofiber mesh reduce the porosity, thus may 365 hinder cell migration. Skin dermis consists of randomly oriented nanofibers (38). 
366 Increasing the fiber diameter and nanofiber orientation may not provide adequate 367 biochemical and biophysical cues needed by the cells to form tissue with proper 368 architecture resembling the native skin.

369

370 Skin mainly consists of keratinocytes in the epidermis and fibroblasts in the dermis. The

371 interaction between these cells is very important in the maintenance of tissue homeostasis

372 and regeneration. Upon injury, cross-talk between keratinocytes and fibroblasts in a 373 double paracrine manner has been found to regulate wound healing. For example,

374 keratinocytes produce IL-1 which stimulates GM-CSF and KGF (that regulates the 375 keratinocyte proliferation and differentiation) production by fibroblasts via the activation 376 of IL-1R (39). In addition, keratinocyte-fibroblast interaction also influences the

377 fibroblast phenotypical changes (fibroblasts to myofibroblasts) and extracellular matrix 378 protein synthesis $(40,41)$. The influences of keratinocytes on fibroblasts' activities in the 379 fibrin and collagen constructs are clearly elucidated in this study. From the results, we 380 found that keratinocyte-fibroblast interaction altered the cell proliferation, contraction and 381 elastic modulus of collagen constructs. The collagen constructs with keratinocytes 382 showed lower cell number, contraction and elastic modulus regardless of the presence of 383 nanofibers. We speculated that decreased cell number created lower contraction force 384 which reduced the contraction and mechanical strength of these constructs. For the fibrin 385 constructs, keratinocyte-fibroblast co-culture increased the cell number. However, 386 contrary to collagen constructs, higher cell number in fibrin constructs did not induced gel 387 contraction and improved the mechanical property. These discrepancies revealed that 388 influence of keratinocytes on fibroblasts cultured in 3-D lattice was greatly affected by 
389 the matrix proteins, as the initial modulus, fibrous network, fibril structure and 390 bioactivities varies from one material to another. In this study, fibrin showed stiff fibril

391 morphology and lower mechanical strength due to different gelation mechanism 392 compared to collagen.

393

394 Uniquely, this study used three non-destructive monitoring techniques, ball indentation, 395 OCT and cell proliferation, to continuously examine the same sample for prolonged 396 culture period, which eliminated the sample preparation variation and enabled 397 identification of the turning point in the cellular activities.

398

399 In summary, we showed that keratinocytes can regulate the fibroblasts' proliferation and 400 fibroblast-mediated gel contraction in 3-D constructs. This regulation is greatly 401 influenced by the matrix proteins, probably via the alternation of keratinocyte soluble 402 factor secretory profile. Reinforcement with nanofibers in collagen and fibrin constructs 403 slightly improved the mechanical property and fibroblast behavior in fibrin.

404

405 Acknowledgement

406 Competing interests: None declared

407 Ethical approval: Not required

408

409

410

411 
412 References

413 [1] Chapekar MS. Tissue engineering: challenges and opportunities. J Biomed Mater Res 2000;53(6):617-620.

415 [2] Nurzynska D, Iruegas M-EP, Castaldo C, Müller-Best P, Di Meglio F. Application 416 of biotechnology in myocardial regeneration-tissue engineering triad: cells, scaffolds, and signaling molecules. BioMed Res Int 2013;2013:236893.

418 [3] MacNeil S. Progress and opportunities for tissue-engineered skin. Nature 419 2007;445(7130):874-880.

420 [4] Sun J, Tan H. Alginate-based biomaterials for regenerative medicine applications. Materials 2013;6(4):1285-1309.

422 [5] Mano J, Silva G, Azevedo HS, Malafaya P, Sousa R, Silva S, Boesel L, Oliveira JM, Santos T, Marques A. Natural origin biodegradable systems in tissue engineering and regenerative medicine: present status and some moving trends. $\mathrm{J}$ R Soc Interface 2007;4(17):999-1030.

426

[6] Lee KY, Mooney DJ. Hydrogels for tissue engineering. Chem Rev 2001;101(7):1869-1880.

428 [7] Drury JL, Mooney DJ. Hydrogels for tissue engineering: scaffold design variables and applications. Biomaterials 2003;24(24):4337-4351.

430 [8] Talwar HS, Griffiths CE, Fisher GJ, Hamilton TA, Voorhees JJ. Reduced type I 431 and type III procollagens in photodamaged adult human skin. J Invest Dermatol 1995;105(2):285-290.

433 [9] Gelse K, Pöschl E, Aigner T. Collagens--structure, function, and biosynthesis. 434 Adv Drug Deliv Rev 2003;55(12):1531-1546. 
435 [10] Gorgieva S, Kokol V. Collagen-vs. gelatine-based biomaterials and their 436 biocompatibility: review and perspectives. 1st ed. Croatia: InTech; 2011.

437 [11] Shoulders MD, Raines RT. Collagen structure and stability. Annu Rev Biochem $438 \quad$ 2009;78:929-958.

439 [12] Nair LS, Laurencin CT. Biodegradable polymers as biomaterials. Progress in 440 Polymer Science 2007;32(8):762-798.

441 [13] Laurens N, Koolwijk P, De Maat M. Fibrin structure and wound healing. J 442 Thromb Haemost 2006;4(5):932-939.

443 [14] Clark RA, Lanigan JM, DellaPelle P, Manseau E, Dvorak HF, Colvin RB. 444 Fibronectin and fibrin provide a provisional matrix for epidermal cell migration 445 during wound reepithelialization. J Invest Dermatol 1982;79(5):264-269.

446 [15] Currie LJ, Sharpe JR, Martin R. The use of fibrin glue in skin grafts and tissue447 448 engineered skin replacements: a review. Plast Reconstr Surg 2001;108(6):17131726.

449 [16] Drew AF, Liu H, Davidson JM, Daugherty CC, Degen JL. Wound-healing defects $450 \quad$ in mice lacking fibrinogen. Blood 2001;97(12):3691-3698.

451 [17] Ahmann KA, Weinbaum JS, Johnson SL, Tranquillo RT. Fibrin degradation 452 enhances vascular smooth muscle cell proliferation and matrix deposition in fibrin-based tissue constructs fabricated in vitro. Tissue Eng Part A 454 2010;16(10):3261-3270.

455 [18] Dvorak HF, Harvey V, Estrella P, Brown L, McDonagh J, Dvorak A. Fibrin 456 containing gels induce angiogenesis. Implications for tumor stroma generation and 457 wound healing. Lab Invest 1987;57(6):673-686. 
458 [19] Gray AJ, Bishop JE, Reeves JT, Mecham RP, Laurent GJ. Partially degraded 459 fibrin (ogen) stimulates fibroblast proliferation in vitro. Am J Respir Cell Mol

460

461

462

463

464

465

466

467

468

469

470

471

472

473

474

475

476

477

478

479 Biol 1995;12(6):684-690.

[20] Moreno-Arotzena O, Meier JG, del Amo C, García-Aznar JM. Characterization of fibrin and collagen gels for engineering wound healing models. Materials 2015;8(4):1636-1651.

[21] Kim B-S, Mooney DJ. Development of biocompatible synthetic extracellular matrices for tissue engineering. Trends Biotechnol 1998;16(5):224-230.

[22] Gunatillake PA, Adhikari R. Biodegradable synthetic polymers for tissue engineering. Eur Cell Mater 2003;5(1):1-16.

[23] Xian LJ, Chowdhury SR, Saim AB, Idrus RBH. Concentration-dependent effect of platelet-rich plasma on keratinocyte and fibroblast wound healing. Cytotherapy 2015;17(3):293-300.

[24] Wimpenny I, Ashammakhi N, Yang Y. Chondrogenic potential of electrospun nanofibres for cartilage tissue engineering. J Tissue Eng Regen Med 2012;6(7):536-549.

[25] Ahearne M, Wilson SL, Liu K-K, Rauz S, El Haj AJ, Yang Y. Influence of cell and collagen concentration on the cell-matrix mechanical relationship in a corneal stroma wound healing model. Exp Eye Res 2010;91(5):584-591.

[26] Wilson SL, Wimpenny I, Ahearne M, Rauz S, El Haj AJ, Yang Y. Chemical and topographical effects on cell differentiation and matrix elasticity in a corneal stromal layer model. Advanced Functional Materials 2012;22(17):3641-3649. 
480 [27] Liu KK, Ju BF. A novel technique for mechanical characterization of thin 481 elastomeric membrane. J Phys D: Appl Phys 2001;34(15):L91.

482 [28] Eisinger M, Sadan S, Silver I, Flick R. Growth regulation of skin cells by 483 epidermal cell-derived factors: implications for wound healing. Proc Natl Acad Sci U S A 1988;85(6):1937-1941.

485

[29] Sese N, Cole M, Tawil B. Proliferation of human keratinocytes and cocultured human keratinocytes and fibroblasts in three-dimensional fibrin constructs. Tissue Eng Part A 2010;17(3-4):429-437.

488

[30] Chakrabarty KH, Heaton M, Dalley AJ, Dawson RA, Freedlander E, Khaw PT, 489 Mac Neil S. Keratinocyte-driven contraction of reconstructed human skin. Wound 490 Repair Regen 2001;9(2):95-106.

491

[31] Isaac C, Paggiaro AO, Aldunate JLCB, Herson MR, Altran SC, Mônica Beatriz M, 492 493 Ferreira MC. Role of keratinocytes in wound contraction: an impact assessment using a model of collagen matrix populated with fibroblasts. Revista Brasileira de Cirurgia Plástica 2011;26(3):402-406.

495 [32] Souren JE, Ponec M, van Wijk R. Contraction of collagen by human fibroblasts 496 and keratinocytes. In Vitro Cell Dev Biol 1989;25(11):1039-1045.

497

[33] Nien Y-D, Han Y-P, Tawil B, Chan LS, Tuan T-L, Garner WL. Fibrinogen 498 499 inhibits fibroblast-mediated contraction of collagen. Wound Repair Regen 2003;11(5):380-385.

500 [34] Zafarulla Khan FK, Shafi HZ, Nufaiei F, Furquan SA, Matin A. Morphology, 501 Mechanical Properties and Surface Characteristics of Electrospun 502 Polyacrylonitrile (PAN) Nanofiber Mats. IJAENT 2015;2(3):15-22. 
503 [35] Meng Z, Wang Y, Ma C, Zheng W, Li L, Zheng Y. Electrospinning of 504 PLGA/gelatin randomly-oriented and aligned nanofibers as potential scaffold in 505 tissue engineering. Mater Sci Eng C Mater Biol Appl 2010;30(8):1204-1210.

506 [36] Yin Z, Chen X, Chen JL, Shen WL, Nguyen TMH, Gao L, Ouyang HW. The 507 collagen expression in dermal fibroblasts. J Invest Dermatol 2008;129(2):340-347. 


\section{Figure legends}

527

528 Figure 1 Mechanical testing. (A) Schematic diagram of the ball indentation setup to

529 measure mechanical property. (B) Illustration of construct indentation by a ball 530 (Reproduced with permission from Royal Society Publishing).

532 Figure 2 Murine dermal fibroblast and epidermal keratinocyte cultures. (A) Co533 culture at day 2. (B) Co-culture at day 8. (C) Passage 1 fibroblasts (Day 7). (D) Passage 1 534 keratinocytes (Day 8).

Figure 3 Appearance of collagen (A) and fibrin (B) constructs.

Figure 4 Confocal images showing cell distribution within the collagen and fibrin

540 red fluorescent cells, respectively. Blue, green and red lines represent the $\mathrm{x}, \mathrm{y}$ and z-axis,

541 respectively. (A) $\mathrm{NF}^{-} \mathrm{K}^{+}$collagen; (B) $\mathrm{NF}^{-} \mathrm{K}^{+}$fibrin. (Scale in $\mu \mathrm{m}$ )

543 Figure 5 Changes in cell number with time. The graft shows the absorbance of 544 alamarBlue $^{\circledR}$ at wavelength $590 \mathrm{~nm}$. A higher absorbance corresponds to a higher 545 metabolic activity, which is an indication of higher cell number. (A) Collagen and fibrin 546 constructs without nanofibers. *, significant higher compared to the $\mathrm{NF}^{-} \mathrm{K}^{-}$fibrin 547 construct. ${ }^{+}$, significant higher compared to the $\mathrm{NF}^{-} \mathrm{K}^{+}$fibrin construct. ${ }^{++}$, significant 548 higher compared to the $\mathrm{NF}^{-} \mathrm{K}^{+}$collagen construct. (B) Collagen and fibrin constructs with 
549 nanofibers. ${ }^{\Delta}$, significant higher compared to the $\mathrm{NF}^{+} \mathrm{K}^{-}$collagen construct. ${ }^{\text {, }}$, significant

550 higher compared to the $\mathrm{NF}^{+} \mathrm{K}^{+}$collagen construct. ${ }^{\Delta \Delta}$, significant higher compared to the $551 \quad \mathrm{NF}^{+} \mathrm{K}^{-}$fibrin construct.

552

553 Figure 6 Changes in construct thickness along culture time. (A) Collagen and fibrin 554 constructs without nanofibers. *, significant different compared to the $\mathrm{NF}^{-} \mathrm{K}^{-}$and $\mathrm{NF}^{-} \mathrm{K}^{+}$ 555 fibrin constructs. ${ }^{+}$, significant different compared to the $\mathrm{NF}^{-} \mathrm{K}^{+}$fibrin construct. **, 556 significant different compared to all the other constructs. ${ }^{++}$, significant different 557 compared to the $\mathrm{NF}^{-} \mathrm{K}^{-}$and $\mathrm{NF}^{-} \mathrm{K}^{+}$fibrin constructs. (B) Collagen and fibrin constructs 558 with nanofibers. ${ }^{\Delta}$, significant different compared to all the other constructs.

559

560 Figure 7 Changes in gel elastic modulus along culture time. (A) Collagen and fibrin 561 constructs without nanofibers. (B) Collagen and fibrin constructs with nanofibers. *, $562 \mathrm{NF}^{-} \mathrm{K}^{-}$and $\mathrm{NF}^{+} \mathrm{K}^{-}$collagen constructs at day 22 demonstrated significantly higher 563 Young's modulus compared to all the earlier time points. 